\title{
Light Hadron Spectrum in Quenched Lattice QCD with Staggered Quarks
}

\author{
Seyong Kim \\ Department of Physics, Sejong University, Seoul 143-747, Korea \\ Shigemi Ohta \\ Institute for Particle and Nuclear Studies, KEK, Tsukuba, 305-0801, Japan
}

\begin{abstract}
Without chiral extrapolation, we achieved a realistic nucleon to $\rho$-meson mass ratio of $m_{N} / m_{\rho}=$ $1.23 \pm 0.04$ (statistical) \pm 0.02 (systematic) in our quenched lattice QCD numerical calculation with staggered quarks. The systematic error is mostly from finite-volume effect and the finite-spacing effect is negligible. The flavor symmetry breaking in the pion and $\rho$ meson is no longer visible. The lattice cutoff is set at $3.63 \pm 0.06 \mathrm{GeV}$, the spatial lattice volume is $(2.59 \pm 0.05 \mathrm{fm})^{3}$, and bare quarks mass as low as $4.5 \mathrm{MeV}$ are used. Possible quenched chiral effects in hadron mass are discussed.
\end{abstract}

Reproducing the known light hadron mass spectrum is the most important test the numerical lattice QCD is yet to pass, in spite of the steady progress [] since the pioneering works by Weingarten and Hamber and Parisi []. The main obstacle is the difficulty in including light dynamical quarks, and consequently the available full-QCD calculations still suffer from too heavy quark mass, too coarse lattice spacing or too small lattice volume []. On the other hand, with the quenched approximation where one neglects dynamical quark loops, recent calculations use small enough lattice spacing and large enough lattice volume to understand the systematic errors arising from them []. Indeed recent quenched calculations [] collectively have shown that both of these errors are smaller than the statistical noise, albeit with rather heavy quarks. Yet these calculations left three major problems: nucleon to $\rho$-meson mass ratio is too high, pion to $\rho$-meson mass ratio is too high, and extrapolating the results to more realistically light quark mass values is necessary but difficult because of the subtle issue of the quenched chiral effect []. Hence quenched calculations with realistically light quark mass values on a large enough and fine enough lattice are desirable.

Helped by the results from ref. [], we choose a gauge coupling of $6 / \mathrm{g}^{2}=6.5$ and a lattice volume of $48^{3} \times 64$. We will find later in this letter that these parameters correspond to the lattice spacing of $a=0.0544(9) \mathrm{fm}$ or the cutoff of $a^{-1}=3.63(6) \mathrm{GeV}$ and a spatial volume of $(2.59(5) \mathrm{fm})^{3}$. We use staggered quarks because it is definitely superior to the Wilson one in controlling the quark mass and hence in investigating the issue of quenched chiral effect: the quark mass is well defined and protected by the remnant $\mathrm{U}(1)$ chiral symmetry in the former while in the latter one encounters a difficult problem of defining the critical hopping parameter under the inevitable presence of exceptional gauge configurations []. For the gauge part we use the single-plaquette Wilson action because our lattice spacing is fine enough.

A combination of multi-hit Metropolis and overrelaxation algorithms is used to generate Monte Carlo samples of quenched gauge field. Separation be- tween propagator sampling is 2000 such updates: 1000 Metropolis interleaved with 1000 over-relaxation. This is proven good enough from the auto-correlation analysis of the obtained pion propagators. We use the conjugate gradient (CG) method for inverting the staggered quark Dirac matrices. A few different sizes of corner and even wall sources with bare mass values of $m_{q} a=(0.05,0.04,0.03,0.02,0.015,0.0075)$ (Set I) and $m_{q} a=(0.01,0.005,0.0025,0.00125)$ (Set II) are used for calculating staggered quark propagators. These two propagator sets are obtained from two almost independent sets of gauge field configurations: they share only a few gauge field configurationsin common. This is to further reduce correlations and should lead us to better comparison of fitting results from one set with those from the other set. They are all combined with point sinks. Set I and Set II together, the bare quark mass varies for over a factor of 40 and provides us a good theater in studying the chiral behaviors. We tried two different kinds of wall definition and a few different source sizes to eliminate systematics arising from using a single kind and size. 118 of quark propagators is collected for Set I, and 250 for Set II. The numerical algorithms are basically the same as in ref. [] and technicalities associated with our implementation on VPP-500 vector-parallel supercomputer is given in ref. []. Preliminary reports of the obtained results were given in ref. [].

Table [1 summarizes our estimates for the pion, $\rho$-meson and nucleon mass values. Here $m_{\pi} a$ is the mass estimate for the mass of Goldstone pion, while $m_{\pi_{2}} a$ is the estimate for non-Goldstone pion extracted simultaneously with the estimate for its parity partner scalar $f_{0} / a_{0}$. Similarly $m_{\rho} a$ is the estimate from the vector meson partnered with $b_{1}$ axial while $m_{\rho_{2}} a$ is the estimate extracted simultaneously with its parity partner $a_{1}$ axial. The nucleon mass $m_{N} a$ is from the even-source results which gave better signals than the corner-source ones. Fitting is done by minimizing the correlated $\chi^{2}$ calculated from a single elimination jack-knife data set. From these data a few immediate conclusions follow: 1) In Fig. 1 we show our Edinburgh plot, $m_{N} / m_{\rho}$ vs. $m_{\pi} / m_{\rho}$. For 
our lightest bare quark mass of $m_{q} a=0.00125$, we get $m_{N} / m_{\rho}=1.230 \pm 0.035$ and $m_{\pi} / m_{\rho}=0.273 \pm 0.006$. The former is in good agreement with the observed value, although the latter is still about $50 \%$ larger. 2) The flavor symmetry breaking estimated by $\left(m_{\pi_{2}} a-m_{\pi} a\right)$ and $\left(m_{\rho_{2}} a-m_{\rho} a\right)$ is generally small. It decreases as we decrease the bare quark mass so much as to be eventually hidden below statistical errors. The symmetry is restored well enough.

Now let us turn our attention to what we can learn about the systematic errors. First, the finite volume effect: from Table we see that $m_{\rho} a$ between $m_{q} a=0.0025$ and $m_{q} a=0.00125$ does not change within statistical error. Assuming, rather safely, that the $\rho$-meson mass dependence on the quark mass is mild in this region around $m_{q} a=0.00125$ and taking the calculated result as physical, we estimate the physical size of our lattice cutoff $a^{-1}$ be $m_{\rho}$ (physical) $/ m_{\rho}\left(m_{q} a=0.00125\right)=$ $0.7700(8) / 0.212(4) \approx 3.63(6) \mathrm{GeV}^{-1}$ or $a=0.0544(9) \mathrm{fm}$. It follows that our lattice has spatial extent of about $2.59(5) \mathrm{fm}$. Using nucleon mass instead of $\rho$-meson mass results in a consistent estimate. It has been argued that the lattice-QCD finite-volume effect is sensitive to the pion Compton wavelength $m_{\pi}^{-1}$ on the lattice: when it is large compared with the lattice, the finite volume effect is expected to fall like $1 / V$ [], and like $\exp \left(-m_{\pi} L\right)$ otherwise []. An extensive study made by the MILC collaboration [] report that the finite-volume effect on a $(2.7 \mathrm{fm})^{3}$ lattice is smaller than $1 \%$. This should translate into at most $1.3 \%$ effect for our lattice volume of $(2.59(5) \mathrm{fm})^{3}$ when $1 / V$ dependence is assumed. With the $\exp \left(-m_{\pi} L\right)$ dependence the effect is smaller than this except for the case of our lightest bare quark mass of $m_{q} a=0.00125$, where the effect is expected to be slightly larger with $m_{\pi} L=2.76(4)$. We also compare our data with existing data at the same $6 / g^{2}=6.5$ but on a smaller $32^{3} \times 64$ lattice [] for $m_{q} a=0.01,0.005$ and 0.0025 . Here we see a $0.7 \pm 1.0 \%\left(m_{q} a=0.01\right)$ to $4.4 \pm 3.2 \%\left(m_{q} a=0.0025\right)$ effect in $m_{\rho} a$ (heavier $m_{\rho} a$ on a larger lattice), and $4.7 \pm 1.0$ $\%\left(m_{q} a=0.01\right)$ to $6.3 \pm 3.2 \%\left(m_{q} a=0.0025\right)$ effect in $m_{N} a$ (lighter $m_{N} a$ on larger lattice). These are consistent with $4.4 \%$ effect expected for the $32^{3}$ volume assuming the $1.3 \%$ effect on the $48^{3}$ volume and the $1 / V$ behavior for $32^{3}$ volume. Therefore we estimate the finite-volume effect in the current nucleon to $\rho$-meson mass ratio $m_{N} / m_{\rho}$ result is $\sqrt{2} \times 1.3 \% \times 1.23 \simeq 2.3 \%$.

Finite-spacing effect: here we expect $O\left(a^{2}\right)$ flavor breaking effect among various definitions of staggered pions and $\rho$ mesons. However as we already discussed, the breaking is hardly visible in our data alone. Comparison with earlier works [] at lower values of $6 / g^{2}(\leq 6.2)$ reinforces this observation. Finite lattice spacing effect in the mass ratio like $m_{N} / m_{\rho}$ should be even smaller than that in $m_{\rho}$ and $m_{N}$ individually. Though there are potential $\mathcal{O}(a)$ effects to $m_{N} / m_{\rho}$ from the flavor symmetry breaking in $m_{\pi} a$ [], it should be negligible as the breaking in $m_{\pi} a$ is already hardly visible.
In addition to these systematic errors mentioned in the above, we considered whether the size of the quark field wall source introduces a systematic bias in choosing the best fit for hadron mass (depending on the size of wall source, excited hadronic states can couple to the wall differently []). For the three different wall sizes, $12^{3}, 24^{3}$, and $32^{3}$, we gathered 300 hadron propagators with $m_{q} a=0.01$ and 124 hadron propagators with $m_{q} a=0.00125$. Fig. 2 shows the effective mass for pion from these different source sizes at $m_{q} a=0.01$. We see an expected tendency that the plateau sets in later for smaller sources. More importantly, within the statistical error they eventually agree with each other and eliminates ambiguity in defining a plateau. Other effective mass plots exhibit the same behavior and help defining plateaus. Thus, our choice of the best fit is less biased by a subjective choice of a plateau.

Thus we established a good enough control of the systematic errors arising from the finite volume, finite cutoff, and choice of plateau in the effective mass, to start discussing the quenched chiral log problem. In Fig. 3, $m_{\pi}^{2} / m_{q}$ vs. $m_{q} a$ is plotted. The fitting form, $m_{\pi}^{2}=C_{0}+C_{1} m_{q}+C_{2} m_{q}^{2}$, has been tried on Set I and Set II separately with correlations among different $m_{\pi}$ 's included. This form has been suggested by the finite volume effect on the pion mass []. At best we get $C_{0}=$ $(1.9 \pm 0.2) \times 10^{-3}, C_{1}=2.20 \pm 0.02, C_{2}=14.4 \pm 0.2$ with confidence level (C.L.) of $1.05 \times 10^{-5}\left(\chi^{2} /\right.$ d.o.f. $\left.=8.6\right)$ for Set I, and $C_{0}=(1.7 \pm 1.1) \times 10^{-4}, C_{1}=2.51 \pm 0.04, C_{2}=$ $-(0.30 \pm 0.33)$ with C.L. of $2.2 \times 10^{-4}\left(\chi^{2} /\right.$ d.o.f. $\left.=13.6\right)$ for Set II. Neither fit is satisfactory. In particular, the fit to Set I overshoots in the quark mass region covered by Set II. This suggests our data in the small mass region are much less singular than the $1 / m_{q}$ behavior of the finite volume effect. For Set II, a fitting form inspired by the quenched chiral perturbation theory [], $\log m_{\pi}^{2} / m_{q}=c-\delta \log m_{\pi_{2}}^{2}$ has been tried, where the correlations among $m_{\pi}$ and $m_{\pi_{2}}$ have been fully taken into consideration. Fitting all the data from Set II together gives $c=0.815 \pm 0.037$ and $\delta=0.027 \pm 0.010$ with C.L. of $9.7 \times 10^{-4}\left(\chi^{2} /\right.$ d.o.f. $\left.=6.93\right)$. This confidence level or $\chi^{2} /$ d.o.f. is better than that for the fitting form considered in the above but it is still marginal. Fitting $m_{q} a=0.005,0.0025$ and 0.00125 data only gives $c=0.691 \pm 0.052, \delta=0.057 \pm 0.013$ with C.L. of $5.8 \times 10^{-2}\left(\chi^{2} /\right.$ d.o.f. $\left.=3.59\right)$ while fitting to the form, $m_{\pi}^{2}=C_{0}+C_{1} m_{q}$, for these three quark masses gives $C_{0}=(2.29 \pm 0.98) \times 10^{-4}, C_{1}=2.47 \pm 0.02$ with C.L. of $1.6 \times 10^{-4}\left(\chi^{2} /\right.$ d.o.f. $\left.=14.2\right)$. One can try to add one more parameter to the above quenched chiral log fit, in order to fit all the data from Set II. Indeed, such a fit gives an improved C.L. $\left(7.0 \times 10^{-3}\right)$ but similar modification (from $C_{0}+C_{1} m_{q}$ to $C_{0}+C_{1} m_{q}+C_{2} m_{q}^{2}$ ) does not improve C.L. of the finite volume fitting form. Thus we think that the quenched chiral fitting form describes our data better and the quenched chiral logarithm behavior discussed in the $m_{\pi}^{2} / m_{q}$ in [] is not a finite lattice volume artifact. 
For nucleon and $\rho$-meson mass values, various fitting forms are suggested and tried in ref. [], where origins of each terms in these fitting forms were discussed. Although these forms should be considered only after the continuum limit is taken, we try these fitting forms on our data since finite lattice spacing effect on our data is small as we discussed in the above. $m_{q}^{1 / 2}$ and $m_{q} \log m_{q}$ terms are from quenched chiral perturbation theory consideration, and $m_{q}$ (from tree level), $m_{q}^{3 / 2}, m_{q}^{2}, m_{q}^{2} \log m_{q}$ (from one loop correction) terms are present both in quenched and ordinary chiral perturbation theory. Following them, we studied chiral extrapolation in $m_{\rho} a$ and $m_{N} a$ using hadron masses from Set I and compared directly with those masses from Set II. Since Set II has four data points, we also tried fits to hadron masses from Set II when the number of fitting parameters is less than four. Correlation among hadron masses is included in the fitting. For Set I, among the twelve fitting forms suggested in ref. [], we can definitely rule out $a+b m_{q}^{1 / 2}$ and $a+b m_{q}$ because the confidence levels are so poor. Fitting to $a+b m_{q}^{1 / 2}+c m_{q}+d m_{q}^{3 / 2}$ and $a+b m_{q}^{1 / 2}+c m_{q}+d m_{q}^{2}$ return either $d$ with error more or less equal to $d$ suggesting that the $\mathrm{d}$ term is not necessary, or a negative $d$. On the other hand, the fit to $a+b m_{q}^{1 / 2}+c m_{q}$ gives a positive value for $b$, which is inconsistent with quenched chiral perturbation theory []. Similarly, fits to $a+b m_{q}+c m_{q}^{3 / 2}+d m_{q}^{2}, a+b m_{q}^{1 / 2}+c m_{q}+d m_{q} \log m_{q}$, and $a+b m_{q}+c m_{q}^{2}+d m_{q}^{2} \log m_{q}$ give either $d$ with error as large as $d$ or larger than $d$ implying that fitting forms of $a+b m_{q}+c m_{q}^{3 / 2}, a+b m_{q}^{1 / 2}+c m_{q}$, and $a+b m_{q}+c m_{q}^{2}$ are preferred, or a negative value for $d$. All the fits to $a+b m_{q}+c m_{q}^{3 / 2}$ and $a+b m_{q}^{1 / 2}+c m_{q}$ returns a negative $c$. The fit to $a+b m_{q}+c m_{q} \log m_{q}$ gives a negative $c$. The confidence level of the fit to $a+b m_{q}+c m_{q}^{2} \log m_{q}$ is $7.7 \times 10^{-4}$ for $m_{N} a$ and $1.3 \times 10^{-2}$ for $m_{\rho} a$. None of these extrapolations agrees well with results from Set II. In contrast, all the fitting forms except for the $a+b m_{q}$ to Set II give high C.L., telling us that the associated statistical errors of Set II are too large. Following ref. [], we also tried fitting $m_{N}+\lambda_{N} m_{\pi_{2}}$ and $m_{\rho}+\lambda_{\rho} m_{\pi_{2}}$ to $a+b m_{q}+c m_{q}^{3 / 2}+d m_{q}^{2}$ or $a+b m_{q}+c m_{q}^{3 / 2}$ for various $\lambda$ 's but none of them improves confidence level significantly.

In Fig. 4, our $m_{N} a$ and $m_{\rho} a$ are plotted against $m_{\pi_{2}}$. The leftmost circles show expected $m_{N} a$ and $m_{\rho} a$ for physical quark mass which is deduced by the nucleon mass and the pion mass from experiment and by the lattice spacing, $a$, obtained in the above. Quenched chiral perturbation theory suggests a linear dependence on $m_{\pi_{2}}$ with a negative coefficient. A fit gives a positive coefficient with poor confidence level, $\mathcal{O}\left(10^{-4}\right)$ although the fit looks good to eyes. Our data in Fig. 4 clearly show that the coefficient of linear term is small, in agreement with small $\delta(0.02 \sim 0.06)$ from our $m_{\pi}^{2} / m_{q}$ data. The smallness of the coefficient for the linear $m_{\pi_{2}}$ term and the disagreement between the extrapolated results from Set I and the actual Set II data, lead us to conclude that one needs very high statistics to trust chiral extrapolation of hadron mass data when lattice calculation is performed with heavy quark masses in order to obtain a result similar to MILC collaboration []. It is interesting that for $m_{q} a<0.005$, the obtained values of pion and $\rho$-meson mass would allow $\rho \rightarrow \pi \pi$ decay. Influence of such decay mode on $\rho$-meson mass needs further investigation in the context of quenched approximation.

In conclusion our numerical calculation of quenched QCD with $(2.59(5) \mathrm{fm})^{3}$ spatial volume and $3.6 \mathrm{GeV}$ cutoff yields a realistic mass ratio of $m_{N} / m_{\rho}=1.230 \pm$ $0.035 \pm 0.023$ at the bare quark mass of $m_{q} a=0.00125$ $\simeq 4.5 \mathrm{MeV}$, where the first error is statistical and the second is finite-volume. Finite lattice spacing effect is negligible. This result is obtained without chiral extrapolation. Flatness of $m_{N} a$ and $m_{\rho} a$ in the region $m_{q} a \leq 0.005$ implies that there will be little variation in lattice simulation of $m_{N} a$ and $m_{\rho} a$ from our lightest quark mass to physical quark mass. Concerned with quenched chiral perturbation behavior, we tried chiral extrapolations for $m_{\pi}, m_{\rho}$ and $m_{N}$ from heavier quark mass $\left(m_{q} a=0.0075 \sim 0.05\right)$ (these fitting forms make sense only in the continuum limit. However, since our lattice spacing is quite small, we tried various fitting forms assuming that there are little modification on the fitting forms due to the finite lattice spacing effect) and compared extrapolated values with our simulated result with lighter quark mass $\left(m_{q} a=0.00125 \sim 0.01\right)$. For $m_{\pi}^{2} / m_{q}$, although a finite lattice volume argument suggests a singular $1 / m_{q}$ behavior, comparison of a fit using $m_{\pi}^{2}$ from heavier quark mass with $m_{\pi}^{2}$ from lighter quark mass shows that $m_{\pi}^{2} / m_{q}$ from lighter quark mass calculation is less singular than $1 / m_{q}$. In nucleon and $\rho$ meson, unlike in pion, it is hard to distinguish by chiral extrapolation the linear dependence on $m_{\pi_{2}}$, a term expected by quenched chiral perturbation theory, because 1) the statistical fluctuation associated with $m_{N} a$ and $m_{\rho} a$ is larger than that with $m_{\pi}$, and 2) the quenched chiral perturbation parameter, $\delta$, appears to be smaller than that in ref. [] probably due to our larger $6 / \mathrm{g}^{2}$. This $6 / g^{2}$ dependence may be understandable since $\delta$ is related to $m_{0}^{2} /\left(4 \pi f_{\pi}^{2}\right)$ [] and the asymptotic scaling of $m_{0}$ can be different from that of $f_{\pi}$.

We would like to thank S. Sharpe and D.K. Sinclair for helpful discussions. We also thank the Institute for Physical and Chemical Research (RIKEN) for the use of its Fujitsu VPP-500/30 vector-parallel super computing system. S.K. would like to thank Prof. E. Goto for the hospitality during his visits to RIKEN. S.O. would like to thank the RIKEN BNL Research Center where he stayed from time to time during the course of this work.

[1] See recent Lattice conference proceeding for the current 
status, e.g., Nucl. Phys. (Proc. Suppl.) 63 (1998).

[2] D. Weingarten, Phy. Lett. 109B, 57 (1982); H. Hamber and G. Parisi, Phys. Rev. Lett. 47, 1792 (1981).

[3] See for example, R.D. Kenway, Proc. Lattice 98 (Boulder, Colorado), eds. T. DeGrand, C. DeTar, R. Sugar and D. Toussaint, Nucl. Phys. B (Proc. Suppl.) 73, 16 (1999).

[4] S. Gottlieb, Nucl. Phys. B (Proc. Suppl.) 53, 155 (1997).

[5] C. Bernard et al., Phys. Rev. Lett. 81, 3087 (1998).

[6] S. Kim and D.K. Sinclair, Phys. Rev. D48, 4408 (1993).

[7] S. Kim and D.K. Sinclair, Phys. Rev. D52, R2614 (1995).

[8] R. Burkhalter et al., Nucl. Phys. B (Proc. Suppl.) 73, 3(1999).

[9] Ideally, the chiral extrapolation should be performed only after the continuum limit is taken. See S.R. Sharpe, Phys. Rev. D41, 3233 (1990); D46, 3146 (1992); C. Bernard and M. Golterman, Phys. Rev. D46, 853 (1992); Nucl. Phys. B (Proc. Suppl.), 26, 360 (1992).

[10] W. Bardeen, et. al., Phys. Rev. D57, 1633 (1998); Nucl. Phys. B (Proc. Suppl.), 63, 141 (1998).

[11] S. Kim and S. Ohta, Nucl. Phys. B (Proc. Suppl.), 42, 920 (1995).

[12] S. Kim and S. Ohta, Nucl. Phys. B (Proc. Suppl.), 47, 350 (1996); op . cit. 54, 199(1997); op. cit. 63, 185 (1998).

[13] M. Fukugita et al., Phys. Lett. B 294, 380 (1992).

[14] M. Lüscher, Nucl. Phys. B354, 531 (1991).

[15] R.D. Mawhinney, Nucl. Phys. B (Proc. Suppl.), 47, 557 (1996).

[16] J.N. Labrenz and S.R. Sharpe, Nucl. Phys. B (Proc. Suppl.) 34, 335 (1994); Phys. Rev. D54, 4595 (1996); M. Booth, G. Chiladze and A. Falk, Phys. Rev. D55, 3092 (1997).

TABLE I. Bare quark mass $m_{q}$ and hadron mass, all in lattice units. Under the staggered quark formalism the Nambu-Goldstone (NG) pion $\pi$ and non-NG pion $\pi_{2}$ split at $O\left(a^{2}\right)$ because of the flavor symmetry breaking, and so do the $\rho$-mesons $\rho$ and $\rho_{2}$, but the effects are now so small and hardly visible. The nucleon is from the even sources which give better signal than the corner ones.

\begin{tabular}{lccccc}
\hline \hline$m_{q} a$ & $m_{\pi} a$ & $m_{\pi_{2}} a$ & $m_{\rho} a$ & $m_{\rho_{2}} a$ & $m_{N} a$ \\
\hline 0.05 & $0.3845(4)$ & $0.3890(4)$ & $0.4196(6)$ & $0.4198(5)$ & $0.637(1)$ \\
0.04 & $0.3363(4)$ & $0.3394(4)$ & $0.3767(6)$ & $0.3770(6)$ & $0.568(1)$ \\
0.03 & $0.2839(4)$ & $0.2868(4)$ & $0.3322(7)$ & $0.3316(7)$ & $0.495(1)$ \\
0.02 & $0.2266(5)$ & $0.2284(5)$ & $0.2882(9)$ & $0.2878(9)$ & $0.418(2)$ \\
0.015 & $0.1959(6)$ & $0.1962(5)$ & $0.2661(10)$ & $0.2664(11)$ & $0.380(2)$ \\
0.01 & $0.1582(5)$ & $0.1577(5)$ & $0.2434(8)$ & $0.2417(9)$ & $0.336(1)$ \\
0.0075 & $0.1377(6)$ & $0.1394(7)$ & $0.2347(16)$ & $0.2367(15)$ & $0.313(3)$ \\
0.005 & $0.1131(6)$ & $0.1121(8)$ & $0.2229(13)$ & $0.2225(13)$ & $0.293(2)$ \\
0.0025 & $0.0811(7)$ & $0.0850(10)$ & $0.2137(22)$ & $0.2122(21)$ & $0.269(3)$ \\
0.00125 & $0.0576(8)$ & $0.0612(29)$ & $0.2122(37)$ & $0.2117(33)$ & $0.261(6)$ \\
\hline \hline
\end{tabular}

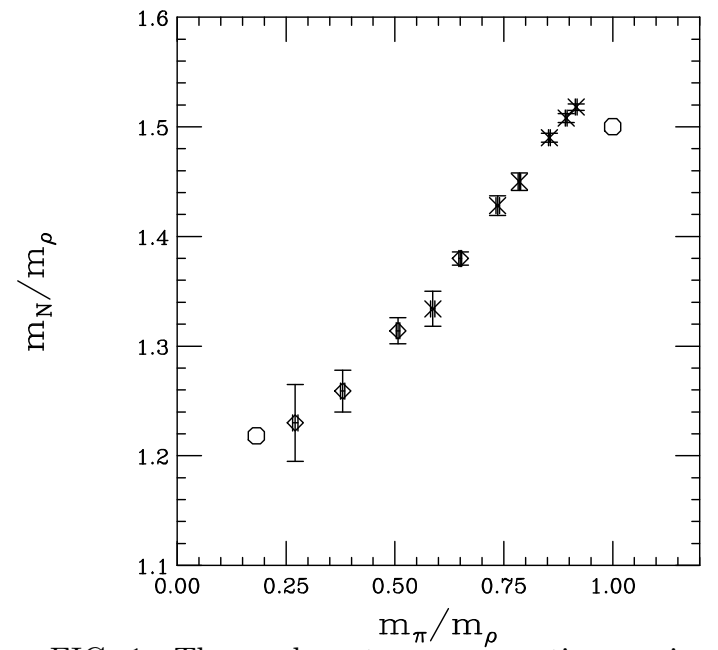

FIG. 1. The nucleon to $\rho$ mass ratio vs. pion to $\rho$ mass ratio at $6 / g^{2}=6.5$ for $m_{q} a=0.05,0.04,0.03,0.02,0.015$, $0.01,0.0075,0.005,0.0025$, and 0.00125 . Set I is plotted with diamonds $(\diamond)$, and Set II crosses $(\times)$. Fit values with only statistical errors are shown and no continuum or finite-volume correction is made. The lower circle represents the experimental value $\left(\left(m_{N} / m_{\rho}, m_{\pi} / m_{\rho}\right)=(1.218,0.182)\right)$ and the upper circle represents the non-relativistic limit $(=(1.5,1.0))$.

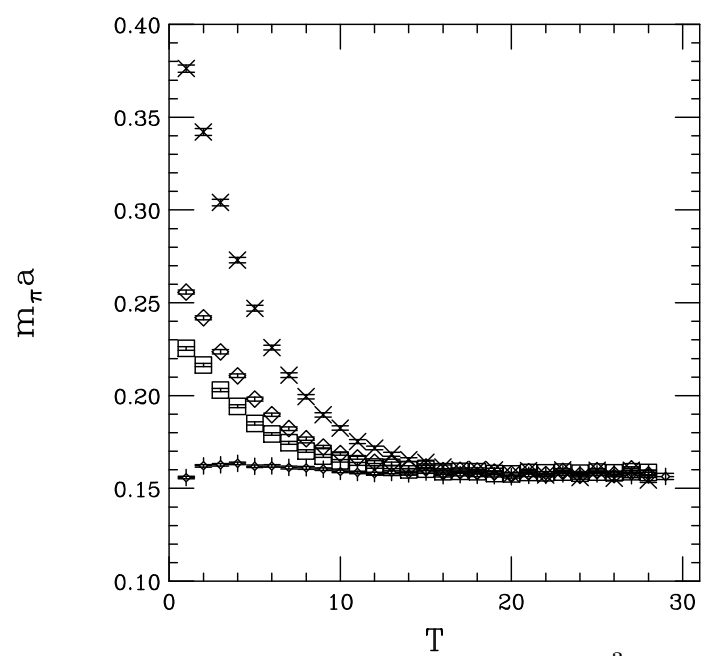

FIG. 2. The pion effective mass from $12^{3}(\times), 24^{3}(\diamond), 32^{3}$ $(\square)$ and $48^{3}(+)$ wall sources at $m_{q} a=0.01$. We see the quality of our plateau when all the four agrees. 


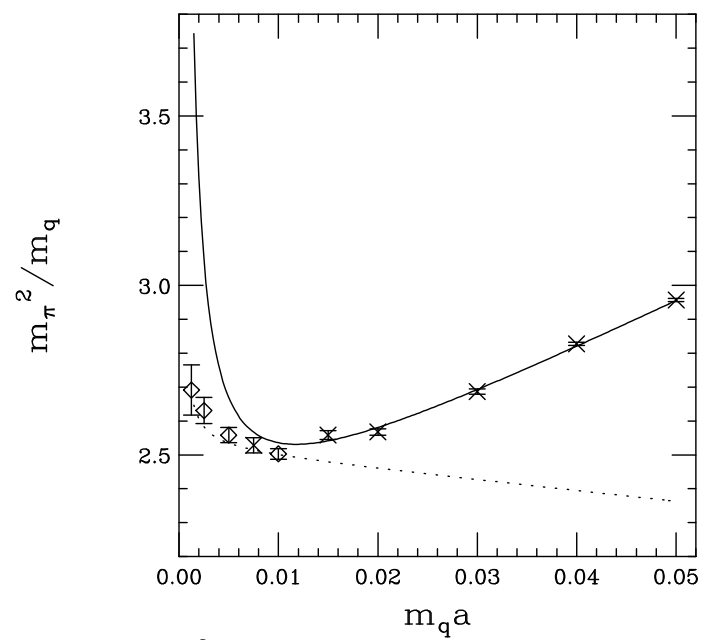

FIG. 3. $m_{\pi}^{2} / m_{q}$ vs. $m_{q} a$. The curves are fit to a form of $m_{\pi}^{2} / m_{q}=C_{0} / m_{q}+C_{1}+C_{2} m_{q}$, to Set I (solid) and Set II (dotted).

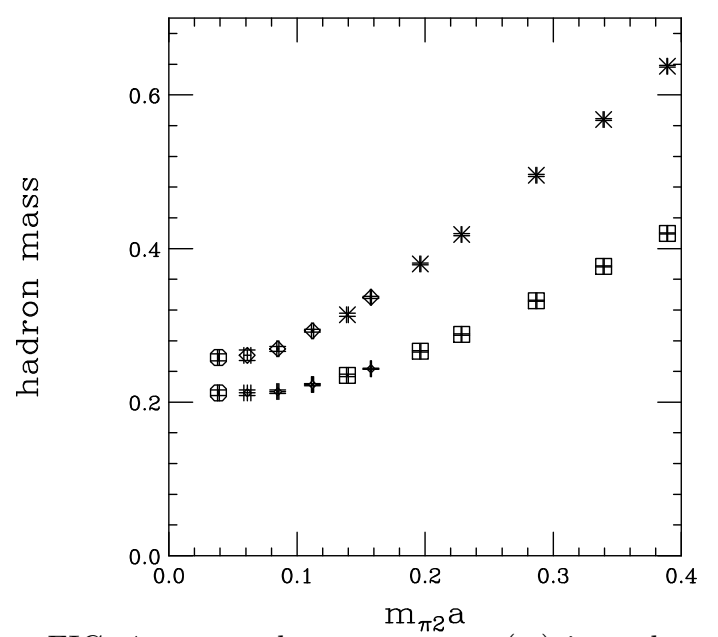

FIG. 4. $m_{N}$ and $m_{\rho}$ vs. $m_{\pi_{2}}$. ( $\left.\times\right)$ is nucleon mass from Set I and $(\diamond)$ is nucleon mass from Set II. ( $\square)$ is $\rho$ mass from Set I and $(+)$ is $\rho$ mass from Set II. The leftmost circles show expected $m_{N} a$ and $m_{\rho} a$ for physical quark mass which is deduced by the nucleon mass and the pion mass from experiment and by the current lattice spacing, $a$. 